\title{
1st-Order Shear Deformable Beam Formulation Based on Meshless Wavelet Galerkin Method
}

\author{
JunHyung Jo ${ }^{1}$ and Yun Lee ${ }^{2}$ \\ ${ }^{1}$ Korea Electric Power Corporation, Daegu Kyungbuk Construction Branch, Gong Pyeung Road 96, Junggu, Daegu, Republic of Korea \\ ${ }^{2}$ Department of Civil Engineering, Daejeon University, 96-3 Yongun-dong, Dong-gu, Daejeon 300-716, Republic of Korea
}

Correspondence should be addressed to Yun Lee; yunis@dju.ac.kr

Received 23 May 2017; Accepted 2 July 2017; Published 27 August 2017

Academic Editor: Damijan Markovic

Copyright (C) 2017 JunHyung Jo and Yun Lee. This is an open access article distributed under the Creative Commons Attribution License, which permits unrestricted use, distribution, and reproduction in any medium, provided the original work is properly cited.

\begin{abstract}
This paper examined and discussed a Meshless Wavelet Galerkin Method (MWGM) formulation for a first-order shear deformable beam, the properties of the MWGM, the differences between the MWGM and EFG, and programming methods for the MWGM. The first-order shear deformable beam (FSDB) consists of a pair of second-order elliptic differential equations. The weak forms of two differential equations are deduced using Hat wavelet series. The exact integration and reduced integration were used to analyze the problems. Some indeterminate beam problems are considered. Condition numbers of the stiffness matrix were analyzed with exact integration and reduced integration for two cases of these problems. Consequently, the results were converged on the analytic solutions. The shear-locking phenomenon also occurred in the MWGM as it occurs in the conventional FEM. The stiffness matrix calculated from the reduced integration causes a similar numerical error to the stiffness matrix calculated from the exact integration in the MWGM. The MWGM showed desirable results in the examples.
\end{abstract}

\section{Introduction}

The aim of this study was to formulate a Meshless Wavelet Galerkin Method (MWGM) to solve a pair of 2nd-order elliptic differential equations, in other words, the Timoshenko beam differential equation using Hat wavelets. Usually the formulation of a MWGM is similar to the conventional formulation of FEM. However, some differences exist and this paper is focused on these differences. Additionally, the property of the 1st-order shear deformable theory, which is the locking phenomenon, is discussed.

Meanwhile, the wavelet theory has evolved over the last few decades. Similar to the Fourier series expansion, wavelets are used to express arbitrary signals. Even though wavelets so far have been used to process signals, they can be used to solve differential equations. Moreover, wavelet numerical methods have been investigated by many researchers in numerical analysis as well as structural analysis fields. For instance, the B-spline wavelet finite element method was developed to analyze vibrations in structures [1]. The wavelet element method was used to combine biorthogonal wavelet systems using the spectral element method [2]. A study that used Daubechies wavelets as the shape functions was performed to solve the Euler beam bending problem [3]. Additionally, a study using B-spline wavelet as the shape functions was conducted [4]. Basu et al. did a study comparing the FEM, BEM, Meshless Method, and Wavelet Methods [5, 6]. Meanwhile, thin plate problems were analyzed with Daubechies wavelets [7]. Recently Daubechies wavelets and DeslauriersDubuc interpolating functions were used to analyze the Euler beam on elastic foundation and thin plate problem [8]. Most of the studies in the literature have been about spline wavelet and Daubechies wavelet.

This study used the Hat wavelet series for expandability and for the ability of inosculation to other numerical methods. The possibility of the inosculation of numerical methods has become important recently because existing methods have already come into wide use such as the standard hFEM, BEM, and Meshless Method. It is better if expansion and inosculation as well as realization are easy. 


\section{Hat Wavelets Series}

2.1. $L^{2}$ Function and the Space $H^{m}(\Omega)$. Usually, the first derivative of the shape functions of FEM must be square integrable function in the Lévesque sense. In most FEMs, approximations of variational boundary-value problems are concerned with the square integral of the first derivative of functions. As shown in Figures 1 and 2, the wavelet series (scaling functions and wavelet functions) belong to $C^{0}(\Omega)$ and $L^{2}(\Omega)$. The first derivatives of the wavelet series are discontinuous but well defined members of $L^{2}(\Omega)$, in other words, those with the property for which $\int f^{2} d x<\infty$ [9].

The definition of Sobolev classes may be summarized as follows. A function $f$ belongs to the Sobolev classes $H^{m}(\Omega)$ if $f$ and all of its partial derivatives of order less than or equal to $m$ ( $m$ being to a nonnegative integer) are members of $L^{2}(\Omega)$. In (1), $m$ usually is set to 1 because the first derivatives of functions are considered in the variational boundary-value problem.

$$
H^{m}(\Omega)=\left\{f \mid f, \frac{\partial f}{\partial x}, \frac{\partial f}{\partial y}, \ldots, \frac{\partial^{m} f}{\partial z^{m}} \in L^{2}(\Omega)\right\} .
$$

With the above considerations, the following properties should be satisfied to use these functions as basic functions for MWGM.

$\varphi$ and $\Phi$ should be sufficiently differentiable and have a square integrable property.

$\varphi$ and $\Phi$ should satisfy the boundary conditions of the problem.

$\varphi$ and $\Phi$ should have a completeness property.

To summarize, $\varphi$ and $\Phi$ should exist in the $H^{1}$-space and have the same continuity requirement.

\subsection{Basic Approximation Functions Based on Wavelets and} Their Construction Method. The basic scaling functions and wavelet functions should be combined linearly to construct the basis functions for the wavelet series analysis as in (2). In a one-dimensional problem domain, the unknown function $u$ can be approximated by a series expansion. Generally, the series expansion can be defined in terms of wavelets as in (2) as follows:

$$
\begin{aligned}
& u(x)= \sum_{k=-\infty}^{\infty} c_{j_{0}}(k) \varphi_{j_{0}, k}(x) \\
&+\sum_{j=j_{0}}^{\infty} \sum_{k=-\infty}^{\infty} d(j, k) \Phi_{j, k}(x) \\
& \text { With } \quad \varphi_{j_{0}, k}(x)=2^{j_{0} / 2} \varphi\left(2^{j_{0} / 2} x-k\right), \\
& \Phi_{j, k}(x)=2^{j_{0} / 2} \Phi\left(2^{j} x-k\right),
\end{aligned}
$$

where $\varphi(x) \in L^{2}(R)$ is the scaling functions; $\Phi(x)$ is the wavelet functions; $c(k)$ and $d(j, k)$ are the coefficients; $j \in Z$ is the scaling parameter; $k \in Z$ is the translation index (controls the spatial location); $j_{0}$ is the starting scale parameter; and $Z$ is the space of all integers.

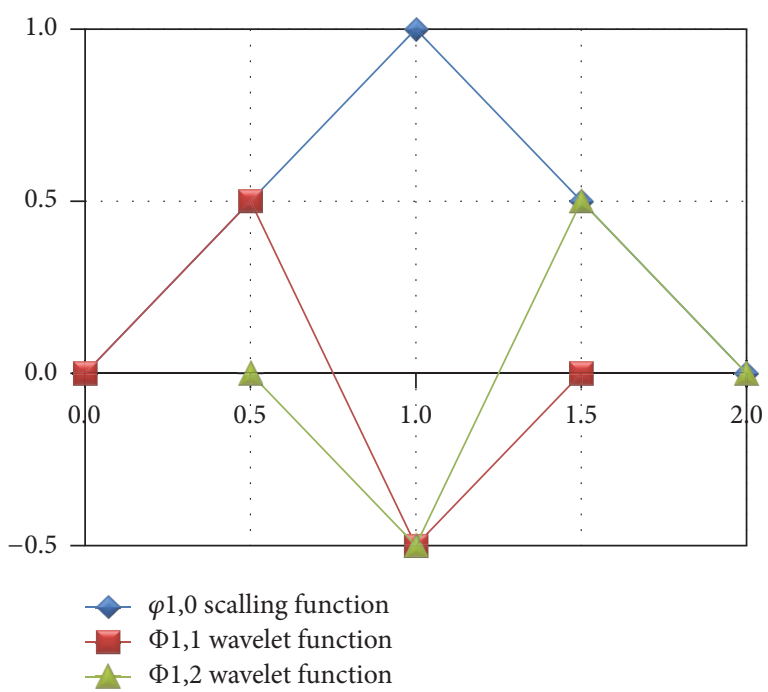

Figure 1: A scaling function $(\varphi)$; wavelet functions $\left(\Phi, V_{1}=V_{0}+W_{0}\right)$.

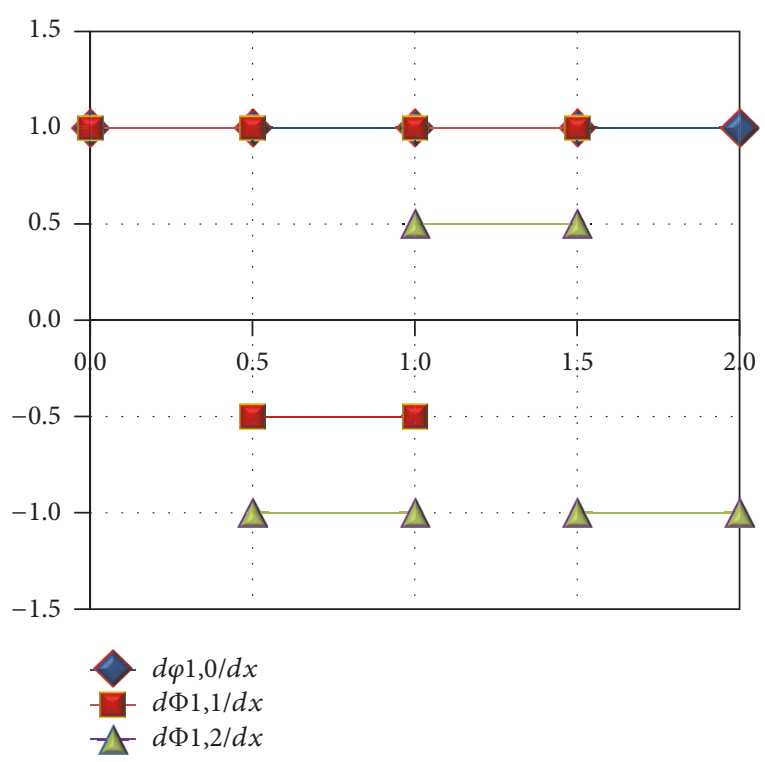

FIGURE 2: Derivatives of a scaling function and wavelet functions.

In the expression for $\varphi_{j_{0}, k}(x)$, the factor $2^{j_{0} / 2}$ helps maintain a constant norm independent of the scale $j_{0}$. The coarsest scale for the space spanned by $\varphi_{k}(x)$ depends on the parameter $j_{0}$. In the proposed research, it is assumed that $j_{0}=0$; that is, the coarsest space is assumed for the initial resolution. This will lead to $\varphi_{j_{0}, k}(x)=\varphi(x-k)$, and (2) becomes

$$
u(x)=\sum_{k=-\infty}^{\infty} c_{k} \varphi_{k}(x-k)+\sum_{j=0}^{\infty} \sum_{k=-\infty}^{\infty} d(j, k) \Phi_{j, k}(x)
$$

The subspace of $L^{2}(R)$ spanned by the scaling functions $\varphi_{k}(x)=\varphi(x-k)$ is obtained as $V_{0}=\overline{\operatorname{Span}_{k}\left\{\varphi_{k}(x)\right\}}$. The different subspaces are related as shown in

$$
V_{0} \subset V_{1} \subset V_{2} \subset V_{3} \subset \cdots \subset L^{2}(R) \quad \text { with } u \in V_{j} .
$$


The next equation is used for approximating signals or functions over the span $(-\infty, \infty)$; thus, the translation factor $k$ is infinity covering all the integers from $-\infty$ and $\infty$ shown in (6). The integer $k$ will be finite to be used in the weak form of the MWGM because the infinity of $k$ cannot be used in the numerical analysis.

$$
u(x)=\sum_{k=-\infty}^{\infty} c_{k} \varphi_{k}(x)+\sum_{j=0}^{\infty} \sum_{k=-\infty}^{\infty} d_{j, k} \Phi_{j, k}(x) .
$$

Wavelet basis functions consist of scaling functions and wavelet functions as mentioned above. The generalized expression of extension is shown in (7) and is used as a refinement scheme. Equation (7) corresponds to the refinement of the mesh for the h-version finite element method.

$$
V_{j+1}=V_{1}+V_{2}+V_{3}+\cdots+W_{j}, \quad j=[0, \infty] .
$$

The following expressions represent examples of the relationship for each level, $j=0,1,2,3$ :

$$
\begin{aligned}
& \text { Level 0: } V_{1}(3)=V_{1}(1)+W_{1}(2) \quad j=0 ; \\
& \begin{aligned}
\text { Level 1: } V_{2}(7)= & V_{0}(1)+W_{0}(2)+W_{1}(4) \\
= & V_{1}(3)+W_{1}(4) \quad j=1 ; \\
\text { Level 2: } V_{3}(15)= & V_{0}(1)+W_{0}(2)+W_{1}(4)+W_{2}(8) \\
= & V_{2}(7)+W_{2}(8) \quad j=2 ; \\
\text { Level 3: } V_{4}(31)= & V_{0}(1)+W_{0}(2)+W_{1}(4)+W_{2}(8) \\
& +W_{3}(16)=V_{3}(15)+W_{3}(16)
\end{aligned} \quad j=3 .
\end{aligned}
$$

The extension of the spaces shows that the spaces are hierarchical, and the refinement can be done by using a suitable value for $j$. If $j$ is taken to be $\infty$, the results of the analysis are exact solutions.

2.3. The Property of the Hat Wavelet Series. There are many wavelet series including the Daubechies, Trigonometric, Hermite Cubic, Haar, and Hat. Because of its suitable properties, this study used the Hat wavelet series as basis functions for the numerical analysis of the weak form of the differential equation, in other words, test functions and trial functions.

Hat wavelet functions have a continuity property but not an orthonormality. After differentiation, the Hat wavelet functions are discontinuous and have a jump property. However the wavelet functions can be integrable after differentiation. The first derivative of the Hat wavelet functions is square integrable. This property is suitable for basis functions for the weak form of the 2 nd-order elliptic differential equation. Equations (9) (12) represent the Hat wavelet functions. They are continuous but do not form an orthonormal basis. Figure 1 shows the relatively simple Hat wavelet functions, which are constructed with (9) and (11) and are used as the basis functions of the MWGM. Usually, (9) is called basic scaling functions and (11) is called wavelet functions.
Basic scaling functions (in $V_{0}$ ) are

$$
\varphi_{0}(x)= \begin{cases}x, & x \in[0,1] \\ 2-x, & x \in[1,2] \\ 0, & \text { otherwise }\end{cases}
$$

Wavelet functions (in $W_{0}$ ) are

$$
\begin{gathered}
\Phi_{0}(x)= \begin{cases}-x, & x \in[0,0.5] \\
3 x-2, & x \in[0.5,1] \\
-3 x+4, & x \in[1,1.5] \\
x-2, & x \in[1.5,2] \\
0, & \text { otherwise }\end{cases} \\
\Phi_{1}(x)= \begin{cases}x, & x \in[0,0.5] \\
-2 x+\frac{3}{2}, & x \in[0.5,1] \\
x-\frac{3}{2}, & x \in[1,1.5] \\
0, & \text { otherwise }\end{cases} \\
\Phi_{2}(x)= \begin{cases}x, & x \in[0,0.5] \\
1-x, & x \in[0.5,1] \\
0, & \text { otherwise. }\end{cases}
\end{gathered}
$$

\section{The Weak Form of the First-Order Shear Deformable Beam Using Hat Wavelets}

The first-order shear deformable beam (FSDB) consists of a pair of second-order elliptic differential equations (13). Each differential equation consists of two independent variables $w$ and $\Psi$, where $w$ is the transverse function and $\Psi$ is the rotation function. The rotation of the $y$-axis is denoted by an independent function $\Psi$. The governing equilibrium equations of the FSDB, namely, the Timoshenko beam theory, are shown by

$$
\begin{aligned}
\frac{d}{d x}\left[G A K_{s}\left(\Psi+\frac{d w}{d x}\right)\right]+f=0, & \Omega \in[0, L], \\
\frac{d}{d x}\left(E I \frac{d \Psi}{d x}\right)+G A K_{s}\left(\Psi+\frac{d w}{d x}\right)=0, & \Omega \in[0, L],
\end{aligned}
$$

where $E$ is the modulus of elasticity, $I$ is the moment of inertia, $G$ is the shear modulus, and $K_{s}$ is the shear correction coefficient.

The weak forms of two differential equations are deduced by multiplying each differential equation with the test functions $w_{1}$ and $w_{2}[10,11]$. Each of the test functions $w_{1}$ and $w_{2}$ corresponds to $w$ and $\Psi$. The test functions of the weak forms belong to $C^{0}(\Omega)$ and exist in $L^{2}(\Omega)$; consequently, the abovementioned Hat wavelet functions can be used as the test functions. Moreover, if the Hat wavelet functions can be used 
for the trial functions of $w$ and $\Psi$, the symmetry property of the stiffness matrix still remains in the MWGM as the standard FEM. The weak form over the domain is developed using the usual procedure. Equation (14) is multiplied by each of the weight functions $-w_{1}$ and $-w_{2}$ and integrated over the domain.

$$
0=\int_{0}^{\Omega}-w_{1}\left\{\left[G A K_{s}\left(\Psi+\frac{d w}{d x}\right)\right]+f\right\} d x
$$

0

$$
=\int_{0}^{\Omega}-w_{2}\left\{\frac{d}{d x}\left(E I \frac{d \Psi}{d x}\right)+G A K_{s}\left(\Psi+\frac{d w}{d x}\right)\right\} d x .
$$

It is clear that (14) is as follows:

$$
\begin{aligned}
& \text { find } w \in H^{1} \text { such that } B\left(w_{1}, v_{1}\right)=l\left(v_{1}\right), v_{1} \in H^{1} \\
& \text { find } s \in H^{1} \text { such that } B\left(s_{1}, v_{2}\right)=l\left(v_{2}\right), v_{2} \in H^{1} \text {. }
\end{aligned}
$$

If the integrands are integrable on the problem domain $\Omega$, (16) are obtained by integrating the first of each integral once by parts. The integral domain is taken over $\Omega$ in (14). Generally, after calculating the element stiffness matrices, the assembling procedure of the element stiffness matrices should be performed. However, nonzero values for the scaling functions and wavelet functions exist on several elements continuously in the MWGM. Accordingly, it is very easy to calculate the assembled global stiffness matrix if the integral domain is taken over the whole domain where the problem is defined. And in doing so, the procedures for assembling the element equations, that is, the stiffness matrices and load vectors, can be circumvented. In other words, the reason for not using the element but instead the domain to integrate the equations is that a global stiffness matrix of the problem domain can be calculated without assembling the element stiffness matrices. In fact, only integration sections are used to integrate a square of the first derivatives of the shape functions over the domain in the MWGM instead of the above-mentioned "element." The properties of the sections are discussed next.

$$
\begin{aligned}
0= & \int_{0}^{\Omega}\left\{\frac{d w_{1}}{d x}\left[G A K_{s}\left(\Psi+\frac{d w}{d x}\right)\right]+w_{1} f\right\} d x \\
& -\left[w_{1} G A K_{s}\left(\Psi+\frac{d w}{d x}\right)\right]_{\partial \Omega}, \\
0= & \int_{0}^{\Omega}\left\{\frac{d w_{2}}{d x} E I \frac{d \Psi}{d x}+w_{2} G A K_{s}\left(\Psi+\frac{d w}{d x}\right)\right\} d x \\
& -\left[w_{2} E I \frac{d \Psi}{d x}\right]_{\partial \Omega} .
\end{aligned}
$$

As in (16), second terms of the right hand sides of these equations except for the weight functions $w_{1}$ and $w_{2}$ are the shear force and the bending moment shown in (17) and (18), respectively.

$$
\begin{aligned}
G A K_{s}\left(\Psi+\frac{d w}{d x}\right) & \equiv V, \\
E I \frac{d \Psi}{d x} & \equiv M .
\end{aligned}
$$

Equation (19) is the approximation functions of $w$ and $\Psi$. If the scaling functions and wavelet functions can be considered as the same kind of functions, $w_{1}$ and $w_{2}$ can be concisely written as (20). The forms of (20) are similar to the standard FEM. The numbers $n_{1}$ and $n_{2}$ are the number of polynomial terms. If $n_{1}$ and $n_{2}$ are not equal, consistent interpolation elements appear in the equations. In this case, a reduced integration does not need to be used. If $n_{1}$ and $n_{2}$ are equal, reduced integration elements with equal interpolation appear in the equations. These attributes are equal to the standard FEM.

$$
\begin{aligned}
w_{1}(x) & =\sum_{k=-\infty}^{\infty} c_{k} \varphi_{k}(x)+\sum_{j=0}^{\infty} \sum_{k=-\infty}^{\infty} d_{j, k} \Phi_{j, k}(x), \\
w_{2}(x) & =\sum_{k=-\infty}^{\infty} c_{k} \varphi_{k}(x)+\sum_{j=0}^{\infty} \sum_{k=-\infty}^{\infty} d_{j, k} \Phi_{j, k}(x), \\
w_{1} & =\sum_{i=1}^{n_{1}} w_{i}^{(1)} \chi_{i}^{(1)}(x), \\
w_{2} & =\sum_{i=1}^{n_{2}} w_{i}^{(2)} \chi_{i}^{(2)}(x) .
\end{aligned}
$$

Equation (21) follows typical procedures for developing linear equations of the weak form of the partial differential equation. Some differences exist in (21) because of the shape functions that are used in the MWGM. First, $(21)$ has $[0, L]$ as integral domains. These are due to the property of the scaling functions and wavelet functions. This property is that nonzero values for the scaling functions and wavelet functions exist over sections that are introduced to integrate the equations. The sections should be determined to be small enough to integrate the equation on the whole domain. If the sections are determined to be large, the integration cannot be calculated because the scaling functions and wavelet functions are folded in the sections. Second, the solutions of (21) are not the values for the node but for the coefficients (or parameters) of the approximation functions. This also is due to the property of the scaling functions and wavelet functions. Bases of wavelet functions have coefficients of functions whose locations are not determined as unknowns because they have degree of freedoms of functions without fixed positions. Usually, standard FEM has the discontinuous property of the solution derivative in the boundary of the elements. MWGM has the same property also. The derivatives of solutions in the section boundary have the discontinuous property, in other words, $C_{0}$ continuity, because scaling 
functions and wavelet functions are only piecewise basis functions for the approximation.

$$
\begin{aligned}
& \int_{0}^{\Omega}\left[G A K_{s}\left(\frac{d \chi_{i}^{(1)}}{d x} \frac{d \chi_{j}^{(1)}}{d x}\right) w_{i}^{(1)} w_{j}^{(1)}\right. \\
& \left.+G A K_{s} \frac{d \chi_{i}^{(1)}}{d x} \chi_{i}^{(2)} w_{i}^{(1)} w_{j}^{(2)}\right] d x=\int_{0}^{\Omega}\left(\chi_{i}^{(1)} f\right) \\
& \quad \cdot w_{i}^{(1)} d x+w^{1}(0) Q_{1}^{0}+w^{1}(L) Q_{3}^{L}, \\
& \int_{0}^{\Omega}\left[E I\left(\frac{d \chi_{i}^{(2)}}{d x} \frac{d \chi_{j}^{(2)}}{d x}\right) w_{i}^{(2)} w_{j}^{(2)}\right. \\
& \quad+G A K_{s} \chi_{i}^{(2)} \chi_{j}^{(2)} w_{i}^{(2)} w_{j}^{(2)} \\
& \left.\quad+G A K_{s} \chi_{j}^{(2)} \frac{d \chi_{i}^{(1)}}{d x} w_{i}^{(2)} w_{j}^{(1)}\right] d x=w^{1}(0) Q_{2}^{0} \\
& \quad+w^{1}(L) Q_{4}^{L} .
\end{aligned}
$$

Equation (22) is the equation for the matrix form. Finally, (23)-(25) are obtained, and (26) is the simplified matrix form.

$$
\begin{aligned}
& \int_{0}^{\Omega} w_{i}^{(1)}\left[G A K_{s}\left(\frac{d \chi_{i}^{(1)}}{d x} \frac{d \chi_{j}^{(1)}}{d x}\right) G A K_{s} \frac{d \chi_{i}^{(1)}}{d x}\right. \\
& \left.\cdot \chi_{i}^{(2)}\right] d x\left\{\begin{array}{l}
w_{j}^{(1)} \\
w_{j}^{(2)}
\end{array}\right\} \\
& =w_{i}^{(1)} \int_{0}^{\Omega}\left(\chi_{i}^{(1)} f\right) d x+w_{i}^{(1)} \chi_{i}^{(1)}(0) Q_{1}^{0} \\
& +w_{i}^{(1)} \chi_{i}^{(1)}(L) Q_{3}^{L} \text {, } \\
& \int_{0}^{\Omega} w_{j}^{(2)}\left[G A K_{s} \chi_{j}^{(2)} \frac{d \chi_{i}^{(1)}}{d x} w_{i}^{(2)} w_{j}^{(1)} E I\left(\frac{d \chi_{i}^{(2)}}{d x} \frac{d \chi_{j}^{(2)}}{d x}\right)\right. \\
& \left.\cdot w_{i}^{(2)} w_{j}^{(2)}+G A K_{s} \chi_{i}^{(2)} \chi_{j}^{(2)} w_{i}^{(2)} w_{j}^{(2)}\right] d x\left\{\begin{array}{l}
w_{j}^{(1)} \\
w_{j}^{(2)}
\end{array}\right\} \\
& =w_{i}^{(2)} \chi_{i}^{(2)}(0) Q_{2}^{0}+w_{i}^{(2)} \chi_{i}^{(2)}(L) Q_{4}^{L} \text {, } \\
& K_{i j}^{11}=\int_{0}^{\Omega} G A K_{s} \frac{d \chi_{i}^{(1)}}{d x} \frac{d \chi_{j}^{(1)}}{d x} d x, \\
& K_{i j}^{12}=\int_{0}^{\Omega} G A K_{s} \frac{d \chi_{i}^{(1)}}{d x} \chi_{j}^{(2)} d x \\
& K_{i j}^{22}=\int_{0}^{\Omega} E I \frac{d \chi_{i}^{(2)}}{d x} \frac{d \chi_{j}^{(2)}}{d x}+G A K_{s} \chi_{i}^{(2)} \chi_{j}^{(2)} d x, \\
& {\left[\begin{array}{ll}
{\left[K^{11}\right]} & {\left[K^{12}\right]} \\
{\left[K^{21}\right]} & {\left[K^{22}\right]}
\end{array}\right]\left[\begin{array}{l}
\{w\} \\
\{s\}
\end{array}\right]=\left[\begin{array}{l}
\left\{F^{1}\right\} \\
\left\{F^{2}\right\}
\end{array}\right] .}
\end{aligned}
$$

Integration should be performed with (27) over the whole domain to circumvent the assembly of the section stiffness matrix.

$$
\begin{aligned}
& \left\{\int_{0}^{l_{1}} \Gamma_{1}(x) d x+\int_{l_{1}}^{l_{\ldots}} \Gamma_{1}(x) d x+\cdots+\int_{l_{1}}^{L} \Gamma_{1}(x) d x\right\} \\
& .\left\{\begin{array}{c}
w_{j}^{(1)} \\
w_{j}^{(2)}
\end{array}\right\}=\int_{0}^{l_{1}} \Gamma_{2}(x) d x+\int_{l_{1}}^{l_{\ldots}} \Gamma_{2}(x) d x+\cdots \\
& +\int_{l_{1}}^{L} \Gamma_{2}(x) d x+w_{i}^{(1)} \chi_{i}^{(1)}(0) Q_{1}^{0}+w_{i}^{(1)} \chi_{i}^{(1)}\left(l_{1}\right) \\
& \cdot Q_{1}^{0}+w_{i}^{(1)} \chi_{i}^{(1)}\left(l_{\ldots}\right) Q_{1}^{0}+\cdots+w_{i}^{(1)} \chi_{i}^{(1)}(L) Q_{3}^{L}, \\
& \Gamma_{1}(x)=\left[G A K_{s}\left(\frac{d \chi_{i}^{(1)}}{d x} \frac{d \chi_{j}^{(1)}}{d x}\right) G A K_{s} \frac{d \chi_{i}^{(1)}}{d x} \chi_{i}^{(2)}\right], \\
& \Gamma_{2}(x)=\chi_{i}^{(1)} f .
\end{aligned}
$$

Some sets of functions are introduced to code the above equations. $\Lambda_{1 \Omega}$ and $\Lambda_{2 \Omega}$ are sets of shape functions. If the shape functions and their derivatives are stored like in (31) and (32), the component matrices of the global matrix can be calculated by (33) and (34) without having to use complicated procedures. The symbol $\otimes$ follows the dyadic product rule.

$$
\begin{aligned}
& i, j=\text { The numer of wavelet basis functions }
\end{aligned}
$$

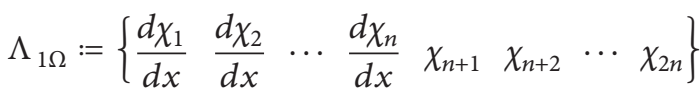

$$
\begin{aligned}
& \Lambda_{2 \Omega}:=\left\{\begin{array}{llllllll}
0_{1} & 0_{2} & \cdots & 0_{n} & \chi_{n+1} & \chi_{n+2} & \cdots & \chi_{2 n}
\end{array}\right\} \\
& K_{1 \Omega}=\int_{0}^{\Omega} \Lambda_{1 \Omega} \otimes \Lambda_{1 \Omega} d x \\
& K_{2 \Omega}=\int_{0}^{\Omega} \Lambda_{2 \Omega} \otimes \Lambda_{2 \Omega} d x \\
& K_{\Omega}=K_{\Omega 1}+K_{\Omega 2} \text {. }
\end{aligned}
$$

\section{The Comparison of the EFG Method and MWGM}

At this point, the similarities and difference of the EFG and MWGM are discussed. As for the similarities, first, the EFG like the MWGM also uses basis functions that exist over the cell which is introduced to integrate the basis functions in the problem domain. Thus, integration and assembly of the stiffness matrix for these two methods can be performed very similarly. As previously stated, sections are introduced to integrate the problem domain in the MWGM. These sections have an effect on the procedures of the MWGM as cells affect the procedures of the EFG. Second, EFG cannot obtain the solutions of the nodes directly; thus, it has to perform additional calculations with the parameters of the basis functions. MWGM also follows the same procedures using the parameters of the basis functions. 
Finally, sections have also a property similar to that of the standard FEM elements, which is a discontinuous attribute in the boundary of the elements. This property stems from the folded shape of the wavelet basis functions. If the folded wavelet basis functions exist in one section, sections as many as wavelet basis functions that are folded should be inserted into a preexisting section to integrate wavelet basis functions. This is equivalent to the mesh refinement of standard FEM and to extending subspaces, which consist of piecewise polynomials.

As for the differences, the EFG performs a Gauss elimination to calculate the basis functions value of the integration points. However, the MWGM does not perform the gauss elimination to obtain the basis functions value of the integration points. The MWGM only follows the method of the standard FEM, which uses simplified procedures for constructing stiffness matrices.

A similar case of sections that are used in this paper is found in [4]. Elements are defined and divided into segments that include nodes in [4]. In other words, the elements are composed of many segments assigned with nodes. Thereafter, the elements are mapped into the standard element domain $\Omega_{s} \in[0,1]$. As a result, many scaling functions and wavelet functions exist in the each elements and the problem domain is the collection of those elements. However, Hat wavelet functions were generated without explicit elements in the problem domain in this paper. The problem domain is composed of the collection of Hat wavelet functions. This is the difference of the way of using basis functions and calculation.

\section{Examples and Discussion}

5.1. The Fixed End Supported Beam Applied by a Dirac Delta Function. Problem domain is

$$
\Omega \in[0,2 L], \quad L=1 .
$$

Governing Equations are

$$
\begin{aligned}
\frac{d}{d x}\left[G A K_{s}\left(\Psi+\frac{d w}{d x}\right)\right]+f & =\delta(x-L) \\
\frac{d}{d x}\left(E I \frac{d \Psi}{d x}\right)+G A K_{s}\left(\Psi+\frac{d w}{d x}\right) & =0 .
\end{aligned}
$$

Boundary Conditions are

$$
\begin{gathered}
w(0)=0, \quad \frac{d w(0)}{d x}=0 \\
w(2 L)=0, \quad \frac{d w(2 L)}{d x}=0 .
\end{gathered}
$$

We considered the indeterminate beam problem shown in Figure 3, which is a fixed end supported beam applied by a Dirac delta function $[12,13]$. The exact integration and reduced integration were used to analyze the problems. Analytic solutions were also obtained for each case. The heights of the beam vary between 0.2 and 1.0. The length of the beam is $2 L(=2.0)$. Figures 4 and 5 show the basis functions of the MWGM. The shear correction factor $k_{s}$ was set to $5 / 6$.

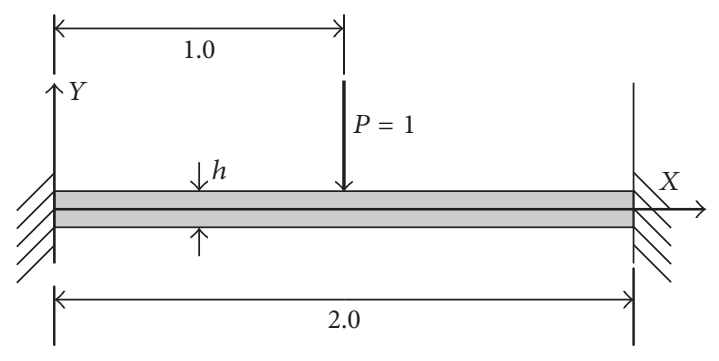

Figure 3: The fixed end supported beam applied by a Dirac delta function.

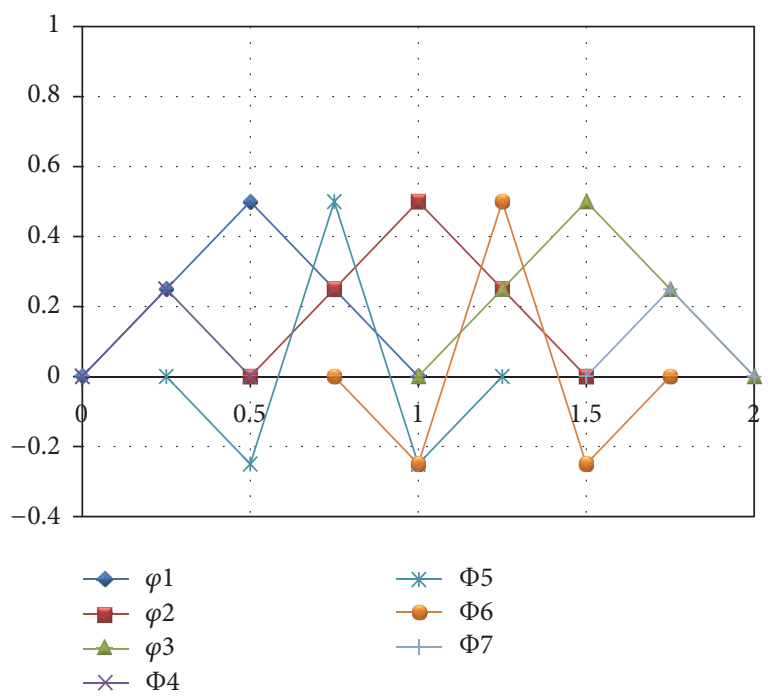

FIGURE 4: 7 functions and 8 sections $(\varphi(x)$ : scaling functions, $\Phi(x)$ : wavelet functions).

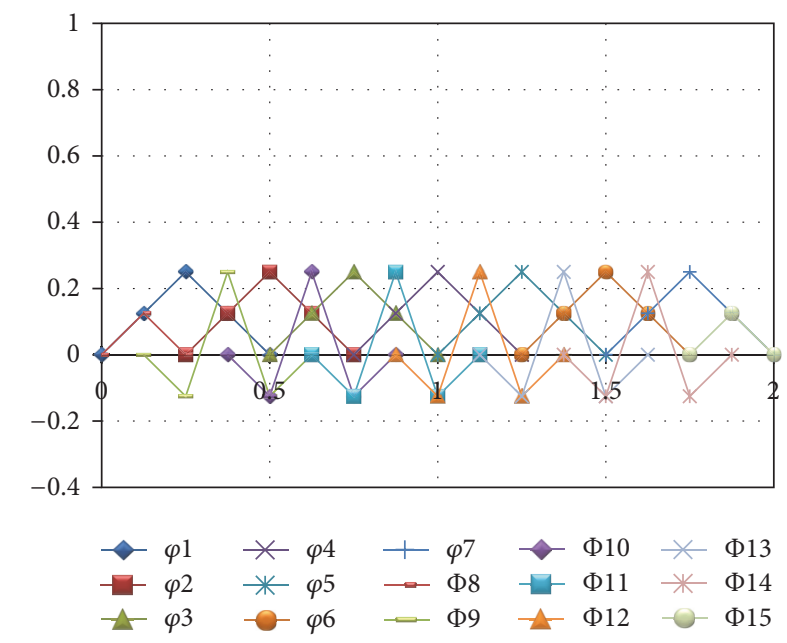

FIGURE 5: 15 functions and 16 sections $(\varphi(x)$ : scaling functions, $\Phi(x)$ : wavelet functions).

As shown in Figure 6, the shear-locking phenomenon definitely occurs in the exact integration when using a small number of functions in the solutions for deflection. As the analyses progress, the shear-locking phenomenon disappears in Figure 6. Figure 6 shows that the reduced integration 


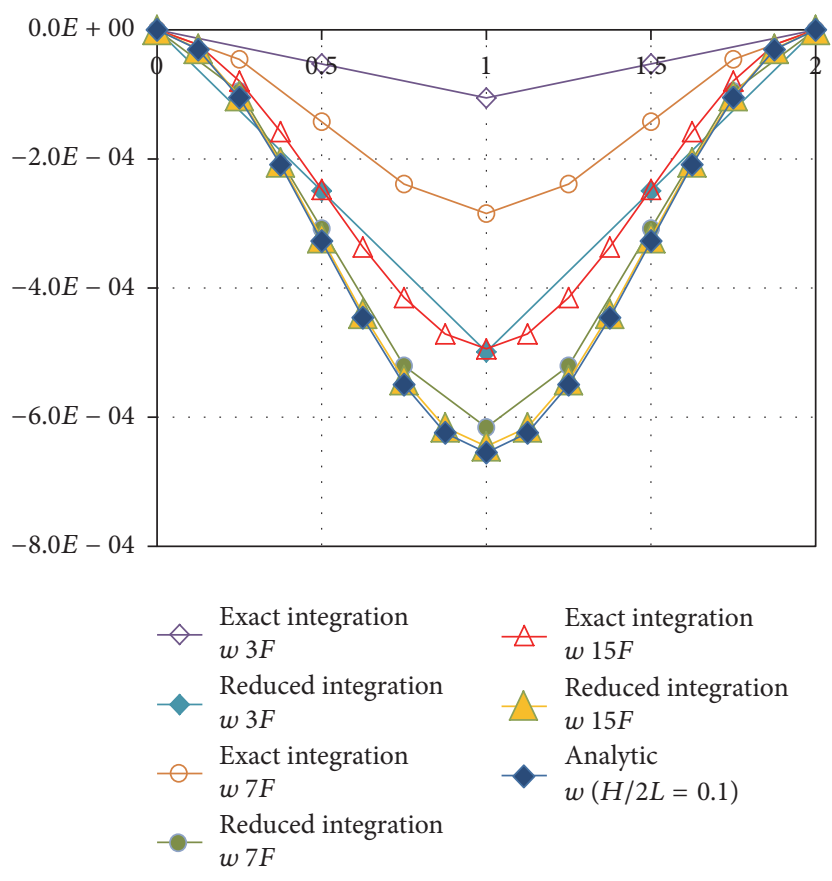

FIGURE 6: Deflection with the exact and reduced integration $(h=$ $0.2, E I=6.667 E-04$, and GAKs $=1.667 E-01)$.

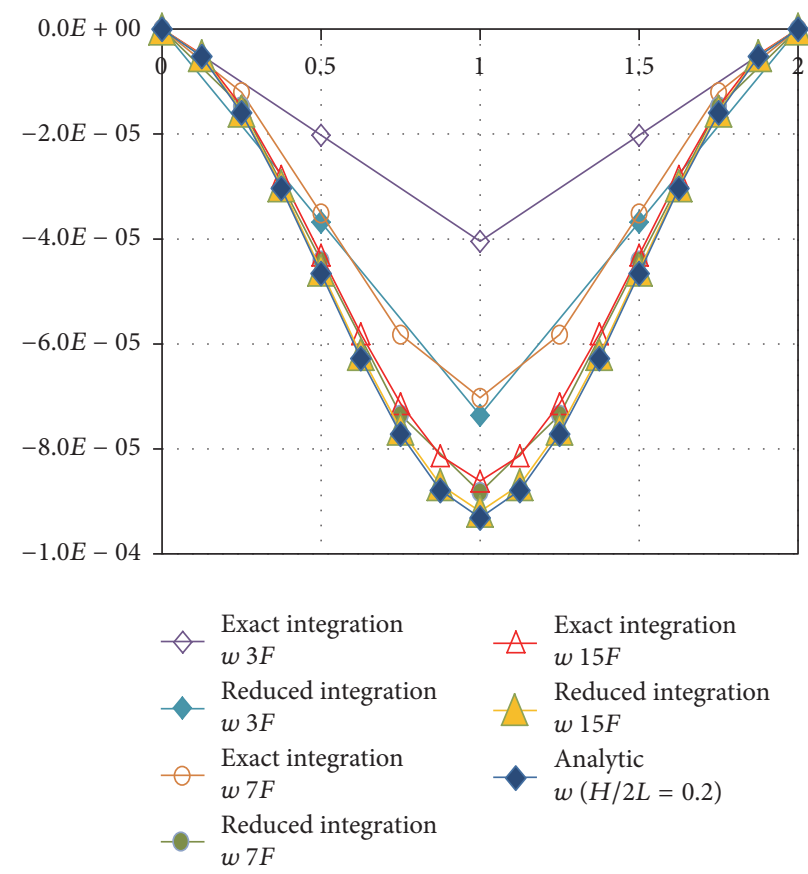

FIgURE 7: Deflection with the exact and reduced integration $(h=$ $0.4, E I=0.533 E-02$, and $\mathrm{GAKs}=3.333 E-01)$.

softens the beam stiffness in the MWGM. As the height of the beam cross section increases progressively from Figures 6-10, the shear-locking phenomenon disappears in the analysis using fewer functions. This is because the deep beam is stiff enough to counteract the shear-locking phenomenon. Although the reduced integration and many functions are used simultaneously, the stiffness of the analysis domain is

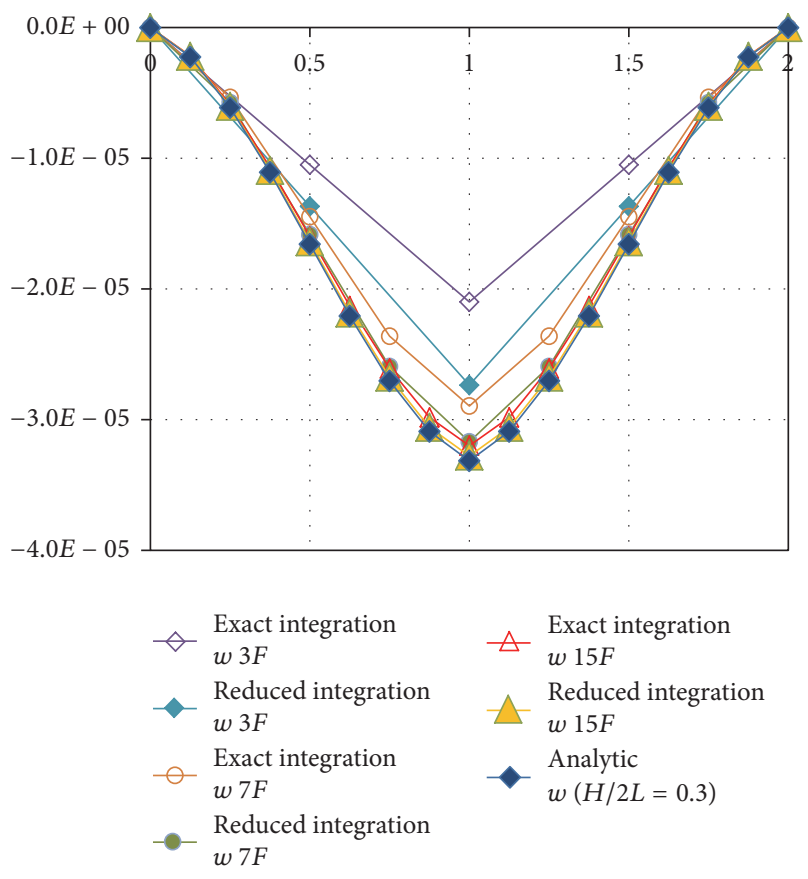

FIGURE 8: Deflection with the exact and reduced integration $(h=$ $0.6, E I=1.800 E-02$, and GAKs $=5.000 E-01)$.

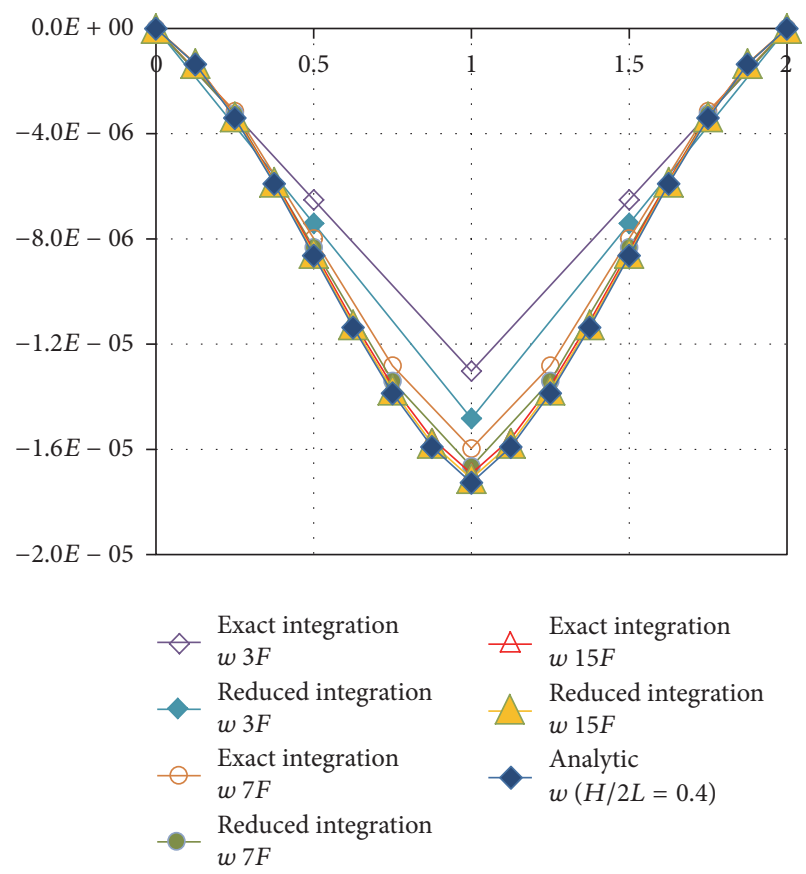

FIgURE 9: Deflection with the exact and reduced integration $(h=$ $0.8, E I=4.267 E-02$, and $\mathrm{GAKs}=6.667 E-01)$.

not overly soft. All the analyses results do not exceed the analytic solution.

As shown in Figure 11, the shear-locking phenomenon definitely occurs in a solution of rotations with exact integration when using a small number of functions. As mentioned above, the solutions of rotations have a similar pattern shown in Figures 6-10. As the analyses progress, the shear-locking 


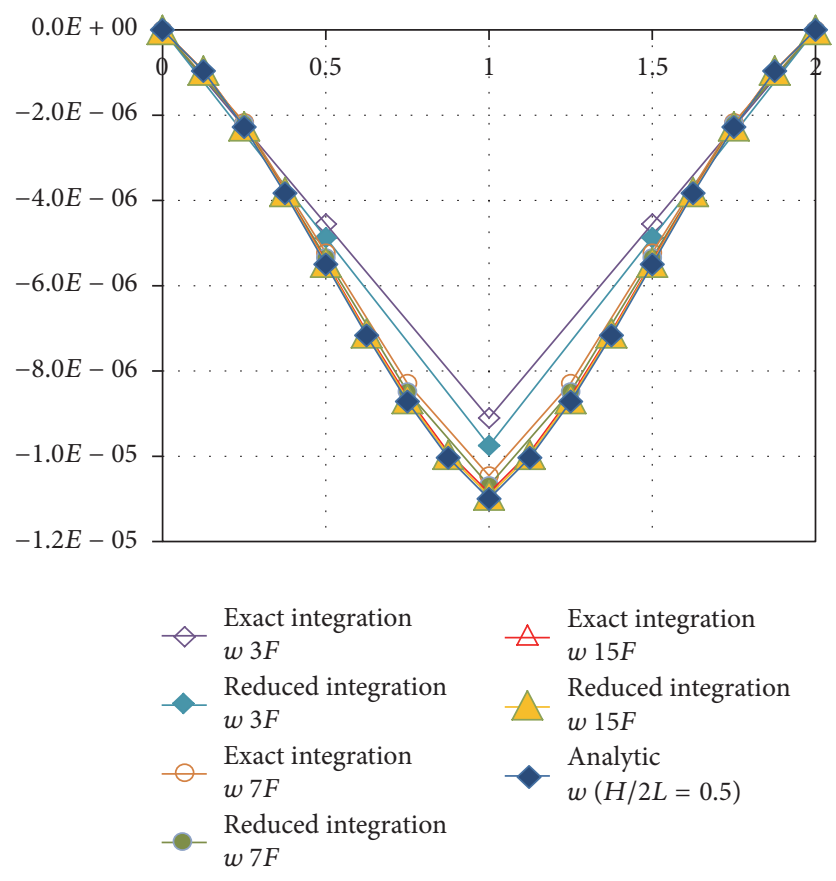

FIGURE 10: Deflection with the exact and reduced integration $(h=$ $1.0, E I=8.333 E-02$, and $\mathrm{GAKs}=8.333 E-01)$.

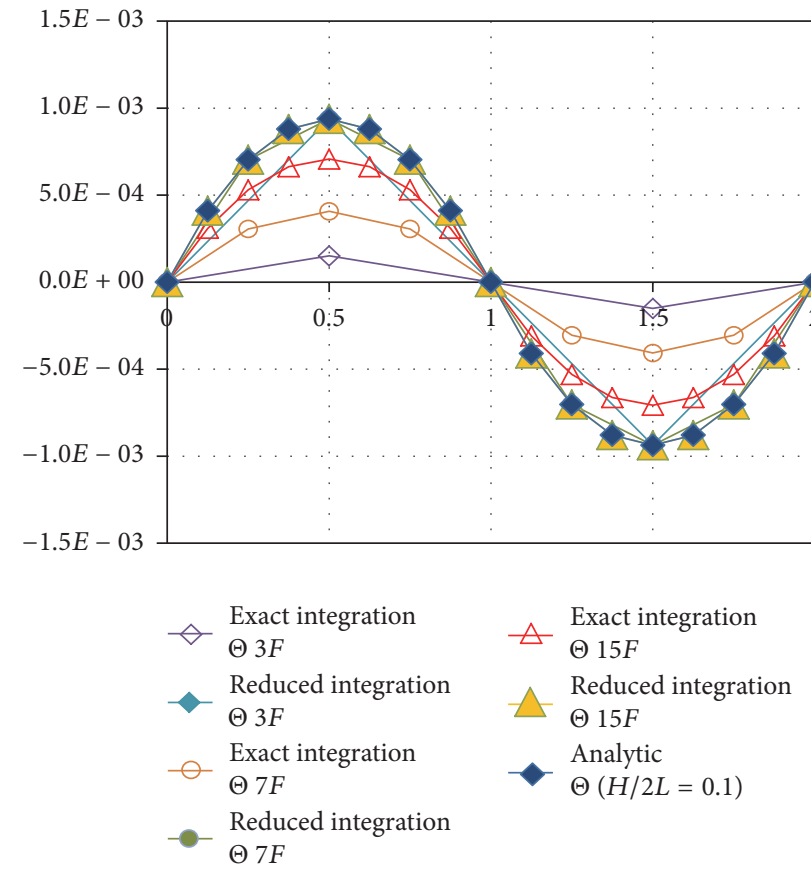

FIGURE 11: Rotations with the exact and reduced integration $(h=0.2$, $E I=6.667 E-04$, and GAKs $=1.667 E-01)$.

phenomenon disappears in Figure 11. Figures 11-15 show the symmetry of the deformed domain. The reduced integration softens the beam stiffness in the MWGM with rotation solutions. It can be seen that everything mentioned above about deflection solutions also apply to rotation solutions.

Additionally, condition numbers of the stiffness matrix were analyzed with exact integration and reduced integration

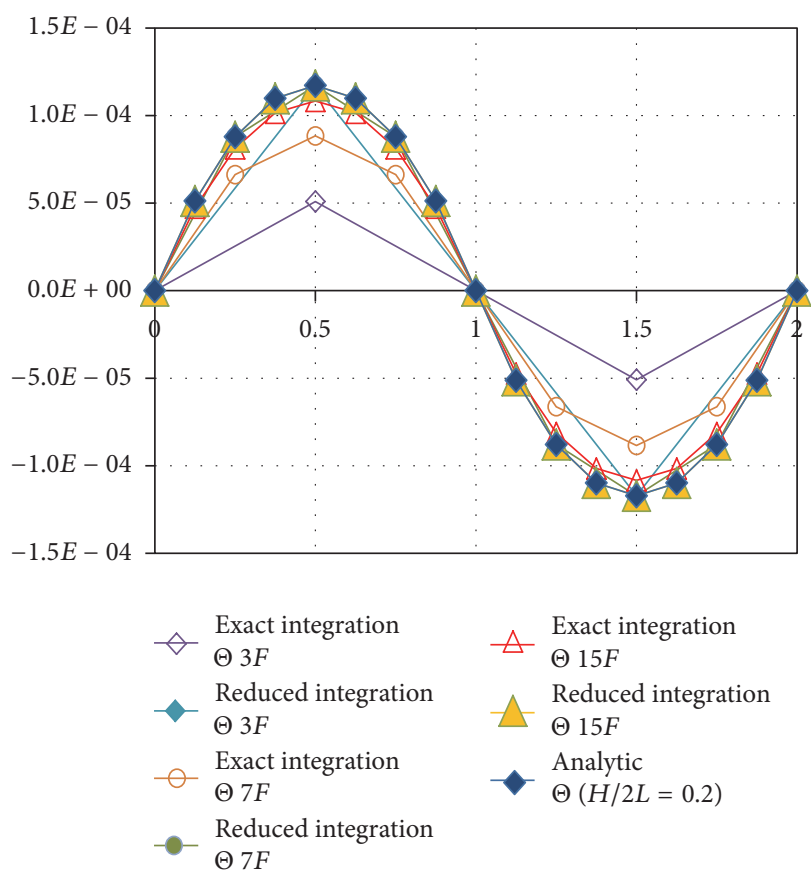

FIGURE 12: Rotations with the exact and reduced integration $(h=$ $0.4, E I=0.533 E-02$, and GAKs $=3.333 E-01)$.

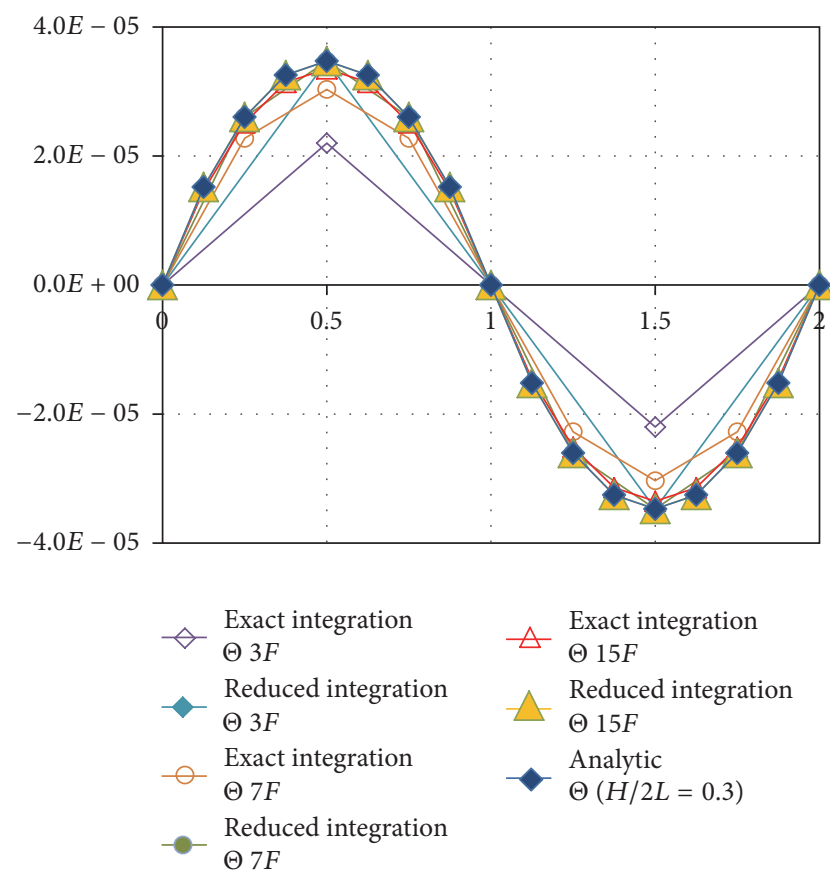

FIGURE 13: Rotations with the exact and reduced integration $(h=$ $0.6, E I=1.800 E-02$, and GAKs $=5.000 E-01)$.

for two cases $(h=0.2$ and $h=0.4)$ in Figures 16 and 17. The condition numbers increase linearly in both cases. Eventually, the ratios of the two values in each case approach 1.0. Thus, the stiffness matrix calculated from the reduced integration causes a similar numerical error as the stiffness matrix calculated from the exact integration in the MWGM. 


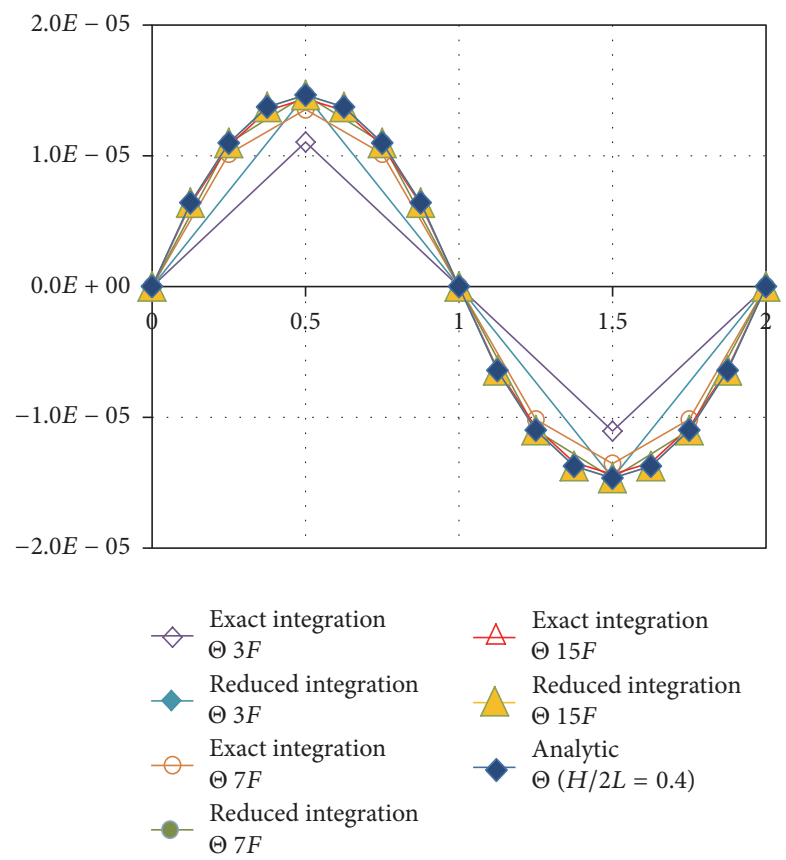

FIGURE 14: Rotations with the exact and reduced integration $(h=$ $0.8, E I=4.267 E-02$, and GAKs $=6.667 E-01$ ).

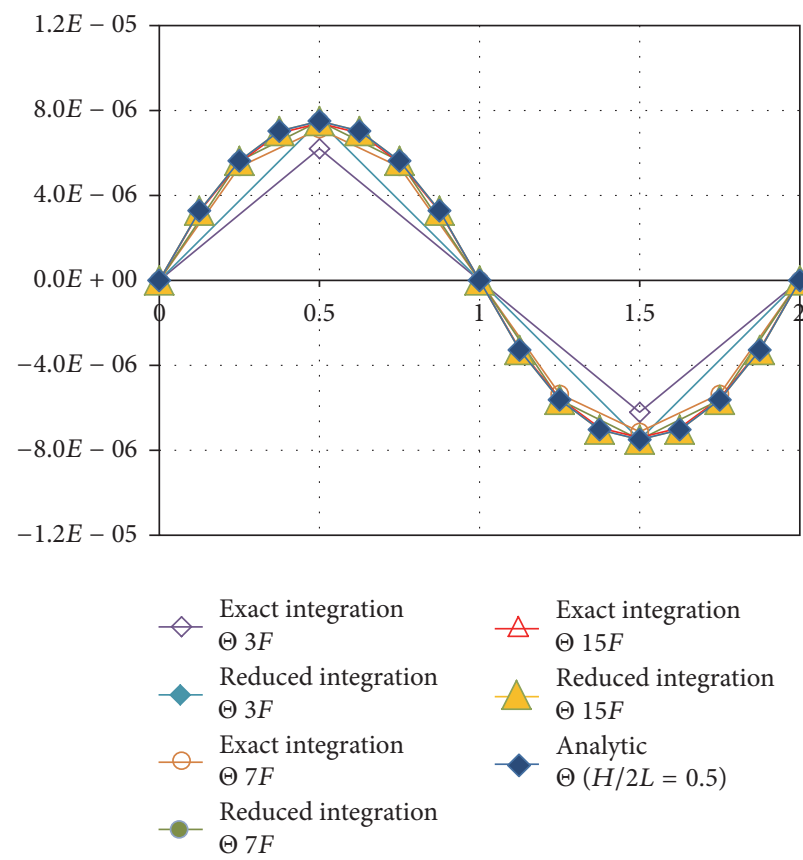

FIGURE 15: Rotations with the exact and reduced integration $(h=$ $1.0, E I=8.333 E-02$, and $\mathrm{GAKs}=8.333 E-01)$.

5.2. The Fixed End Supported Beam with a Nonuniform Section Applied by a Dirac Delta Function. Problem domain and properties of the cross sections are as follows:

$$
\begin{aligned}
\Omega & \in[0,2 L], \quad L=1 \\
x & \in[0,1]: E I_{1}=6.667 E-04, \\
G A K_{s} & =1.667 E-01
\end{aligned}
$$

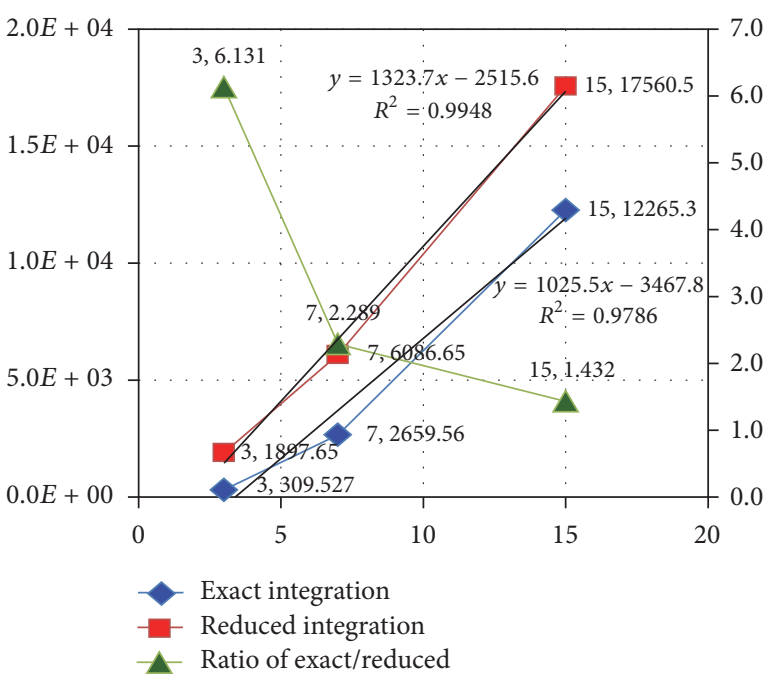

Figure 16: Condition numbers of $[k](h=0.2)$.

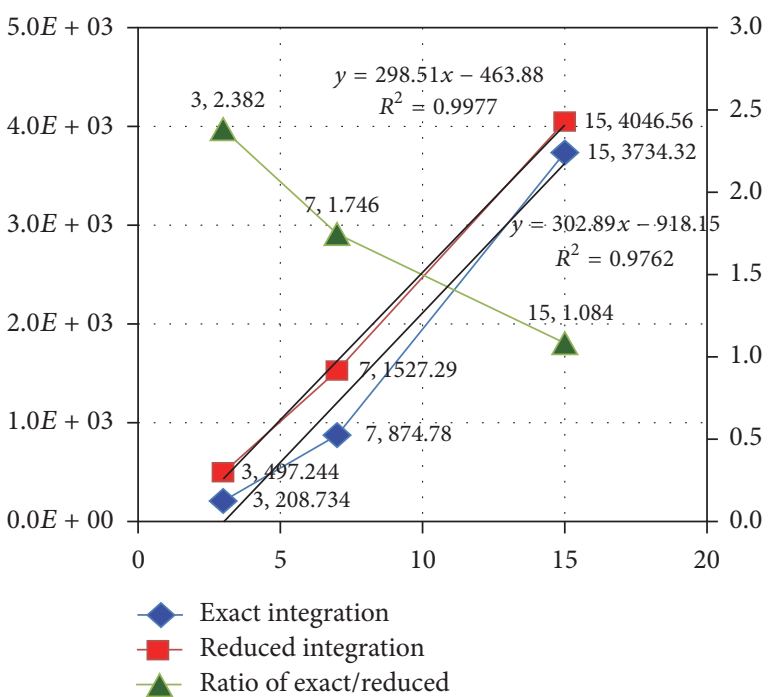

Figure 17: Condition numbers of $[k](h=0.4)$.

$$
\begin{aligned}
x & \in[1,2]: E I_{2}=8 E I=5.334 E-03, \\
G A K_{s} & =-3.5097 E-01 .
\end{aligned}
$$

Governing equations are as follows:

$$
\begin{aligned}
\frac{d}{d x}\left[G A K_{s}\left(\Psi+\frac{d w}{d x}\right)\right]+f & =\delta(x-L) \\
\frac{d}{d x}\left(E I \frac{d \Psi}{d x}\right)+G A K_{s}\left(\Psi+\frac{d w}{d x}\right) & =0 .
\end{aligned}
$$

Boundary conditions are as follows:

$$
\begin{gathered}
w(0)=0, \quad \frac{d w(0)}{d x}=0 \\
w(2 L)=0, \quad \frac{d w(2 L)}{d x}=0 .
\end{gathered}
$$




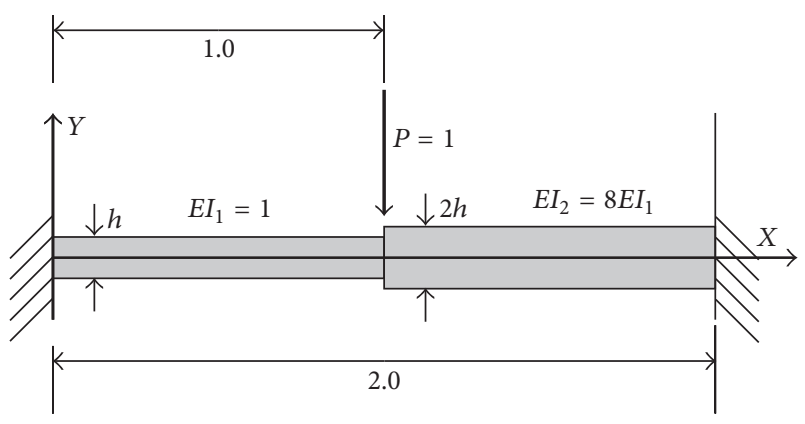

FIGURE 18: The fixed end supported beam with a nonuniform section applied by a Dirac delta function.

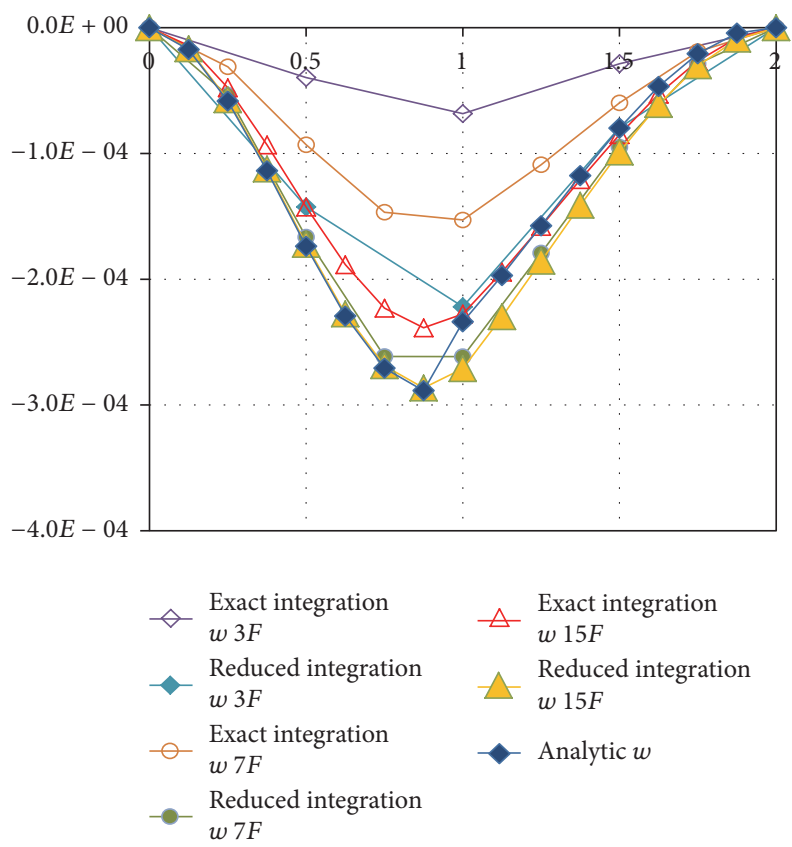

FIGURE 19: Deflection with the exact and reduced integration $(h=$ $0.2,0.4, E I=6.667 E-04, \mathrm{GAKs}=1.667 E-01,8 E I=5.333 E-03$, and GAKs $=-3.509 E-01)$.

Next, we considered the indeterminate beam problem shown in Figure 18, which is a fixed end supported beam consisting of nonuniform sections. Additionally, the Dirac delta function is applied to this beam. The domain of the problem has a jump condition. The analytic solution cannot be obtained in a single domain but in multiple domains. Particularly, the domain $x \in[1,2]$, for which the beam depth is large, has a high ratio of $h / 2 L$. It is difficult to analyze this type of model because of the domain jump condition.

As shown in the MWGM and analytic solution, maximum deflection occurs on the left side of the domain. Figure 19 clearly shows that the maximum deflection locations correspond with each solution. This problem also shows the shear-locking phenomenon, and, as the analyses progress, the shear-locking phenomenon disappears.

Both solutions with the exact integration and reduced integration agree well with each other in the previous problem. However, both solutions do not agree with each other in

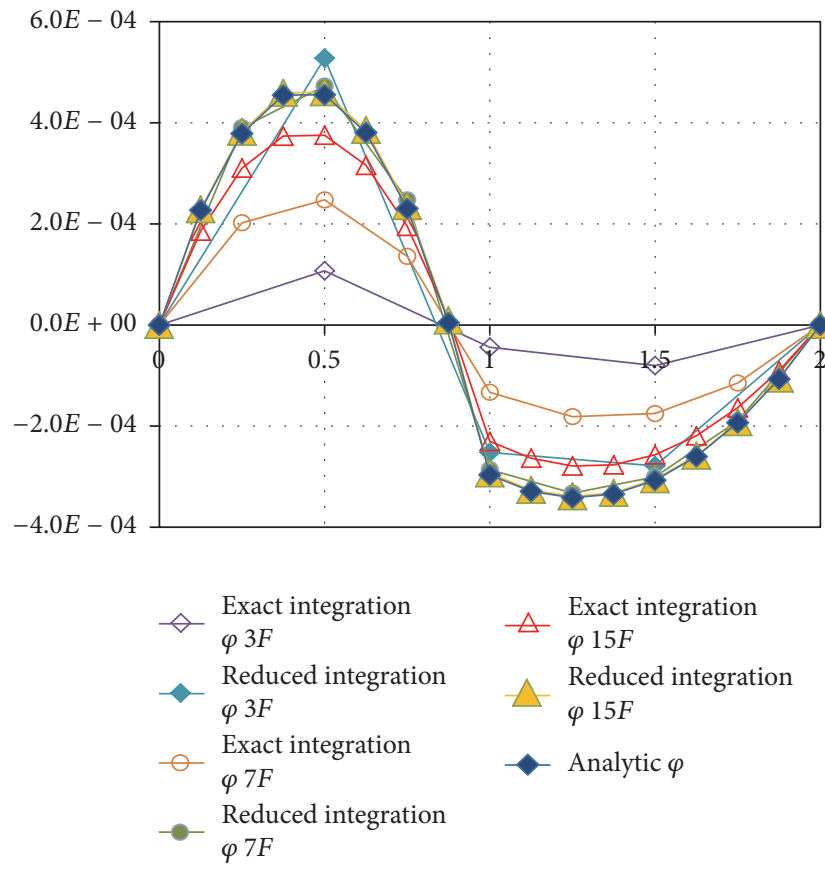

FIGURE 20: Rotations with the exact and reduced integration $(h=$ $0.2,0.4, E I=6.667 E-04, \mathrm{GAKs}=1.667 E-01,8 E I=5.333 E-03$, and GAKs $=-3.509 E-01)$.

this problem. More specifically, the solutions with the exact integration correspond to the analytic solutions in domain $x \in[1,2]$; however, the solutions with the reduced integration correspond to analytic solutions in the domain $x \in[0,1]$. It could be said that the reduced integration is effective for relatively thin beams, and the exact integration is effective for relatively thick beams. The reduced integration solution converges from a large value to a small value, in other words, an analytic solution. However, the exact integration converges from a small value to a large value, in other words, an analytic solution in the rotation solutions (Figure 20).

\section{Conclusion}

This paper examined and discussed the MWGM formulation for a first-order shear deformable beam, the properties of the MWGM, the differences between the MWGM and EFG, and programming methods for the MWGM. The MWGM has also the shear-locking phenomenon. Circumventing assembling the global stiffness matrix $\mathrm{K}$ enables the program to be written easily. Multiresolution and localization analysis, which is similar to adaptive analysis of the finite element method, can be performed with the MWGM. It is carefully predicted that all analysis methods could be used to solve one problem such as structure, fluid, soil, or multiphysics problems in the future. The MWGM could be used as part of the analyses. It is easy to perform multiresolution analysis, in other words, adaptive analysis using the MWGM, because integration and interpolation are separated, and linear functions are used as the bases.

This study used the Hat wavelet series for expandability as well as for the ability of inosculation to other numerical 
methods. As mentioned in the introduction section of this paper, the MWGM is a good alternative and complementary method with traditional and recently developed methods.

\section{Conflicts of Interest}

The authors declare that they have no conflicts of interest.

\section{Acknowledgments}

This research was supported by Basic Science Research Program through the National Research Foundation of Korea (NRF) funded by the Ministry of Education, Science and Technology (NRF-2016R1A2B4016632).

\section{References}

[1] X. Zhang, X. Chen, Z. Yang, B. Li, and Z. He, "A stochastic wavelet finite element method for 1d and 2d structures analysis," Shock and Vibration, vol. 2014, Article ID 104347, 2014.

[2] C. Canuto, A. Tabacco, and K. Urban, "The wavelet element method. I. Construction and analysis," Applied and Computational Harmonic Analysis. Time-Frequency and Time-Scale Analysis, Wavelets, Numerical Algorithms, and Applications, vol. 6, no. 1, pp. 1-52, 1999.

[3] J. Ma, J. Xue, S. Yang, and Z. He, "A study of the construction and application of a Daubechies wavelet-based beam element," Finite Elements in Analysis and Design, vol. 39, no. 10, pp. 965975, 2003.

[4] J. W. Xiang, X. F. Chen, Z. J. He, and H. B. Dong, "The construction of 1D wavelet finite elements for structural analysis," Computational Mechanics, vol. 40, no. 2, pp. 325-339, 2007.

[5] P. K. Basu, A. B. Jorge, S. Badri, and J. Lin, "Higher-order modeling of continua by finite-element, boundary-element, meshless, and wavelet methods," Computers \& Mathematics with Applications, vol. 46, no. 1, pp. 15-33, 2003.

[6] J. H. LIN, Wavelets Modeling in Continuum Mechanics, Thesis Master of Science in civil engineering [M.s. thesis], Vanderbilt University, 1999.

[7] X. Chen, S. Yang, J. Ma, and Z. He, "The construction of wavelet finite element and its application," Finite Elements in Analysis and Design, vol. 40, no. 5-6, pp. 541-554, 2004.

[8] R. B. Burgos, M. A. C. Santos, and R. R. e. Silva, "Analysis of Beams and Thin Plates Using the Wavelet-Galerkin Method," IACSIT International Journal of Engineering and Technology 7 , vol. 7, pp. 261-266, 2015.

[9] E. B. Becker, G. F. Garey, and J. T. Oden, Finte Elements An Introduction Volume 1, Prentice-Hall, 1981.

[10] K.-J. Bathe, "Finite element procedures," Finite element procedures, 1996.

[11] J. N. Reddy, An introduction to the finite element method, McGRAW-HILL, 2nd edition, 1993.

[12] C. E. Augarde and A. J. Deeks, "The use of Timoshenko's exact solution for a cantilever beam in adaptive analysis," Finite Elements in Analysis and Design, vol. 44, no. 9-10, pp. 595-601, 2008.

[13] H. Antes, "Fundamental solution and integral equations for Timoshenko beams," Computers and Structures, vol. 81, no. 6, pp. 383-396, 2003. 


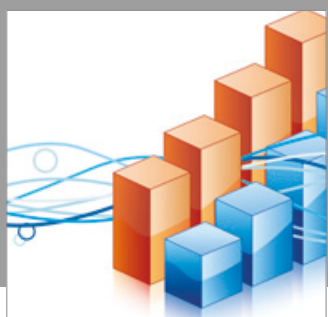

Advances in

Operations Research

vatersals

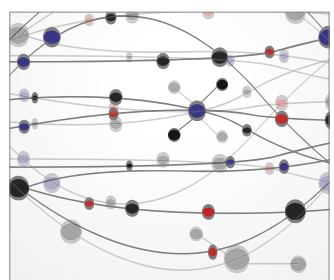

\section{The Scientific} World Journal
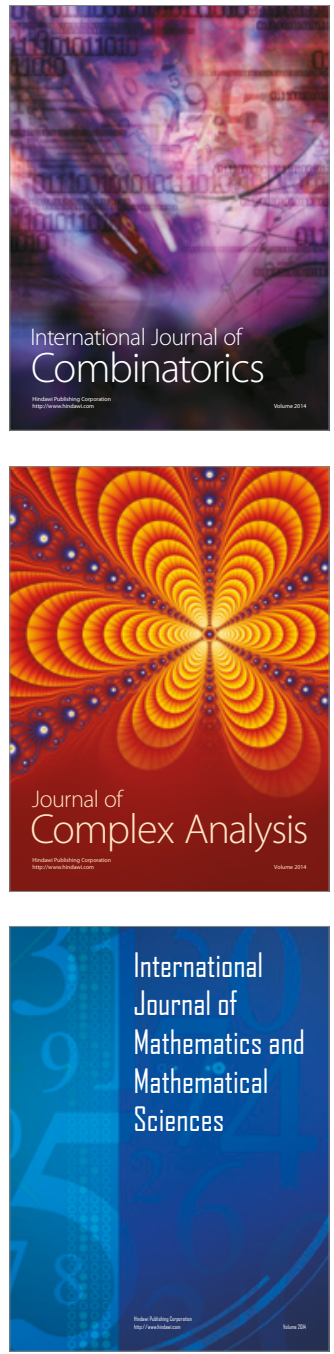
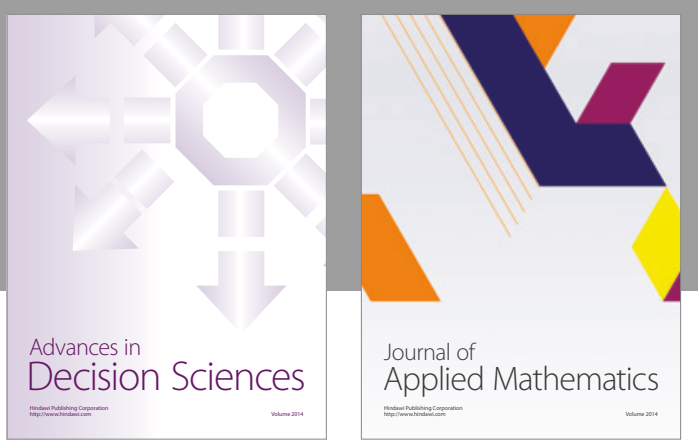

Algebra

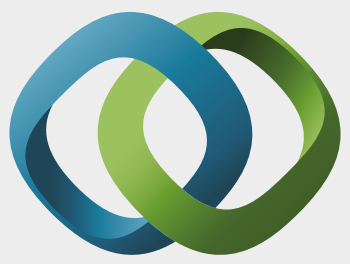

\section{Hindawi}

Submit your manuscripts at

https://www.hindawi.com
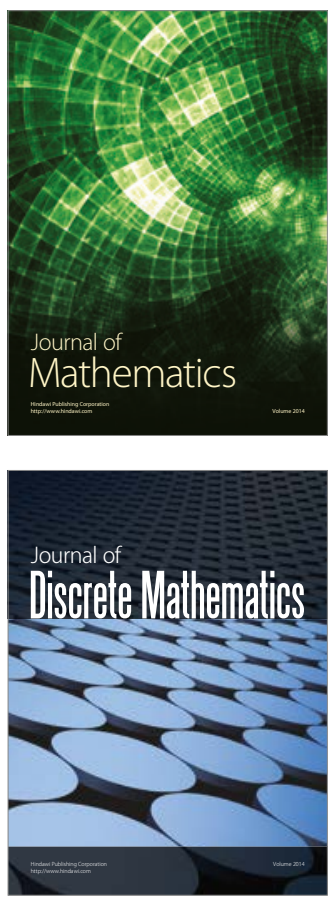

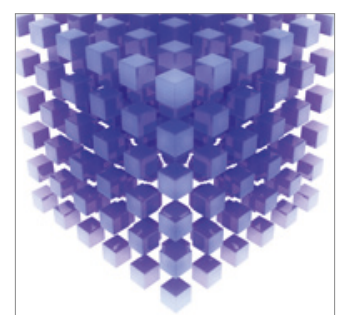

Mathematical Problems in Engineering
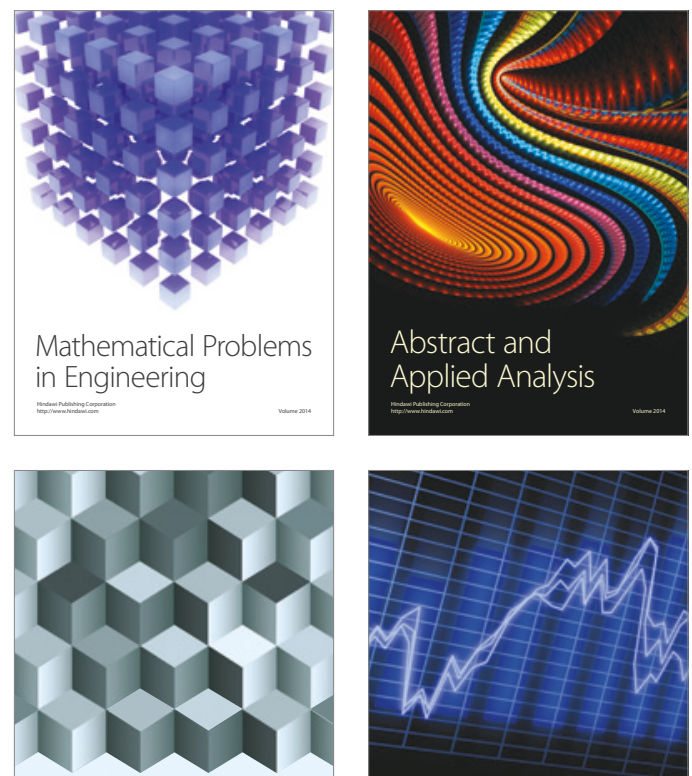

Journal of

Function Spaces

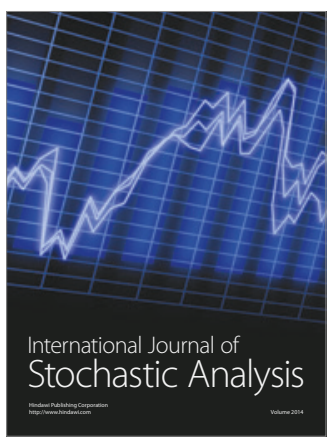

Probability and Statistics
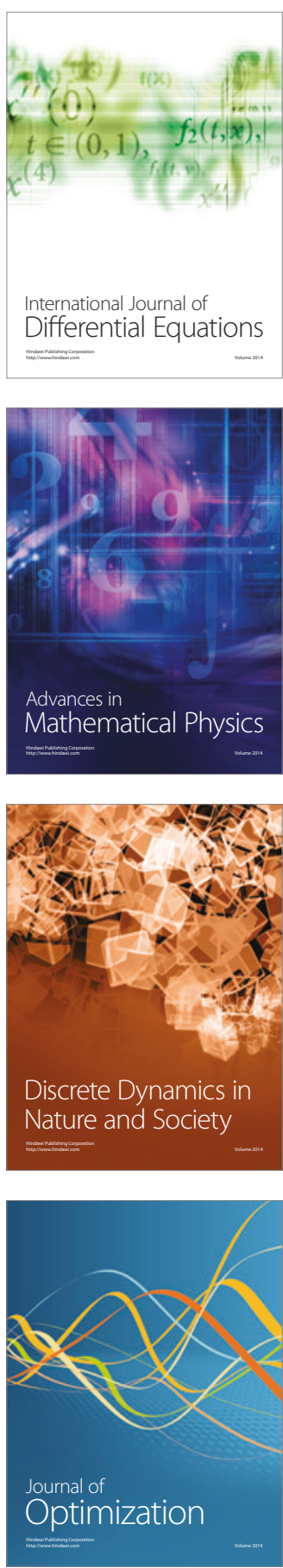